\title{
Extensions of Morphic Quasi-morphic and Centrally Morphic Rings
}

\author{
Serap Şahinkaya
}

(Communicated by Tamer KOŞAN)

\begin{abstract}
In the present paper, we define the ring

$$
T=[R ; I, \sigma, n]:=\left\{a_{0}+a_{1} x+\ldots+a_{n} x^{n} \in \frac{(R, I)[x ; \sigma]}{\left(x^{n+1}\right)}: a_{0} \in R, a_{i} \in I \text { for } i=1, \ldots, n\right\},
$$

which is a subring of $R[x ; \sigma] /\left(x^{n+1}\right)$. It is proved that; If $R$ is a unit regular ring, each $\alpha \in T$ is equivalent to an element $e_{0}+e_{1} x+\cdots+e_{n} x^{n}$, where $e_{0}, e_{1}, \ldots, e_{n}$ is a sequence of orthogonal idempotents such that $e_{0} \in R, e_{1}, \ldots, e_{n} \in I$ and $n \geq 1$. As an application of this, it has shown that;

(1) The ring $[R ; I, \sigma, n]$ is left morphic.

(2) $(R, I)(x) /\left(x^{n+1}\right)$ is left centrally morphic for each $n \geq 0$.

Also, we prove that the ring $(R, I)(x) /\left(x^{n+1}\right)$ is left quasi-morphic.

Keywords: Morphic rings; quasi-morphic rings; unit (strongly) regular rings; centrally morphic rings

AMS Subject Classification (2010): Primary: 16D10
\end{abstract}

\section{Introduction}

Throughout this paper, all rings are associative with identity. For $a, b \in R$, we say that $a$ is equivalent to $b$, if $b=u a v$ for some units $u$ and $v$ in $R$. Let $R$ be a ring. For any element $a$ in $R$, the left (respectively, right) annihilator of $a$ in $R$ is denoted by $\mathbf{l}_{R}(a)$ (respectively, $\mathbf{r}_{R}(a)$ ), and it is well known that $R / \mathbf{l}_{R}(a) \cong R a$ as left $R$-modules.

If $R / R a \cong \mathbf{1}_{R}(a)$, then $a$ is called a left morphic element (see [10]). Equivalently, $a \in R$ is left morphic if and only if there exists $b \in R$ such that $\mathbf{l}_{R}(a)=R b$ and $\mathbf{l}_{R}(b)=R a$ (see [10, Lemma 1]). A ring $R$ is called left morphic if every element of $R$ is left morphic. An element $a$ in $R$ is unit regular if there exists $u \in U(R)$ such that $a=a u a$, where $U(R)$ denotes the group of units of $R$. The ring itself is unit regular if all of its elements are unit regular. $R$ is unit-strongly regular ring, every element $r$ of $R$ there exists a unit element $u \in R, r=r^{2} u$. Ehrlich [3], has shown that a ring is unit regular if and only if it is (von Neumann) regular and left morphic. In [7] Lee and Zhou studied the relationships between these properties for rings of the form $S / I$, where $S$ is either a polynomial ring $R[x]$ or a skew polynomial ring $R[x ; \sigma]$, and where $I$ is an ideal of the form $\left(x^{n}\right)$. Thus by [7, Theorem 2] if $R$ is unit regular, and if the endomorphism $\sigma: R \rightarrow R$ is onto and fixes all idempotents of $R$, then all such rings $S / I$ are left morphic. This had previously been known only when $R$ is strongly regular by [2].

Received : 12-May-2017, Accepted : 26-September-2017 
Let $I$ be an ideal of $R$ and $\sigma: R \rightarrow R$ be a ring endomorphism. Then define ring $T$ as following:

$$
T=[R ; I, \sigma, n]:=\left\{a_{0}+a_{1} x+\ldots+a_{n} x^{n} \in \frac{(R, I)[x ; \sigma]}{\left(x^{n+1}\right)}: a_{0} \in R, a_{i} \in I \text { for } i=1, \ldots, n\right\} .
$$

It is clear that $T$ is a ring (a subring of $R[x ; \sigma] /\left(x^{n+1}\right)$ ). The $\operatorname{ring} S$ below is the special case of $T$ where $\sigma=1_{R}$. Let $\sigma: R \rightarrow R$ is a ring endomorphism such that $\sigma(e)=e$ for all $e^{2}=e \in R$. In section 2, it is shown that $[R ; I, \sigma, n]$ is a left morphic ring for every $n \geq 0$. This result is a generalization of [8, Corollary 3].

The ring $R$ is called left centrally morphic if, for each $a \in R$, there exists $b \in C(R)$ such that $\mathbf{l}_{R}(a)=R b$ and $\mathbf{l}_{R}(b)=R a$, where $C(R)$ denotes the center of $R$ (see [9, Section 5]). In [6], Huang and Chen considered the following situation: Let $I$ be an ideal of a unit regular ring $R$ and let

$$
S:=\frac{(R, I)[x]}{\left(x^{n+1}\right)}=\left\{\sum_{i=0}^{n} a_{i} x_{i}: a_{0} \in R, a_{i} \in I, i=1,2, \cdots, n\right\} .
$$

They showed in [6, Theorem 2.2] that every matrix ring over $S$ is morphic. In [9, Section 5], Lee and Zhou proved that, for an integer $n \geq 1$, a ring $R$ is strongly regular iff $R[x] /\left(x^{n+1}\right)$ is left centrally morphic. Note that this result is a generalization of [7, Corollary 5] and [7, Theorem 12]. In section 3, we generalize this result for the ring $S$.

An element $a \in R$ is called left quasi-morphic if there exist $b, c \in R$ such that $R a=l(b)$ and $R b=l(a)$ (see [1]). This notion was introduced as a generalization of left morphic rings and regular rings. Also in [1], it was shown that left quasi morphic rings share a number of important properties with regular rings. In section 4 , we used the technique which was a generalization of technique that Herbera used in [5]. By using that, we prove that the ring $S$ is quasi morphic for each $n \geq 0$.

\section{The ring $[R ; I, \sigma, n]$ is left morphic}

Proposition 1. [8, Proposition 1] Let $R$ be a semiprime ring and let $\sigma$ be an endomorphism of $R$ such that $\sigma(e)=e$ for all $e^{2}=e \in R$. Then $e\left(\sigma^{k}(x)-x\right)(1-e)=0$ for all $x \in R$, all $e^{2}=e \in R$ and positive integers $k$.

The following Theorem is a generalization of $[8$, Theorem 2$]$. We prove it by the similar way.

Theorem 2. Let $I$ be an ideal of a unit regular ring $R$ and let $\sigma: R \rightarrow R$ is a ring endomorphism such that $\sigma(e)=e$ for all $e^{2}=e \in R$. Then each $\alpha \in T$ is equivalent to $e_{0}+e_{1} x+\cdots+e_{n} x^{n}$, where $e_{0}, e_{1}, \ldots, e_{n}$ is a sequence of orthogonal idempotents such that $e_{0} \in R$ and $e_{1}, \ldots, e_{n} \in I$ and $n \geq 1$.

Proof. It is enough to prove the following claim:

Claim: For each integer $k$, there exists idempotents $e_{0} \in R, e_{1}, \ldots, e_{k-1} \in I$, and $r_{k}, \ldots, r_{n} \in I$ such that up to equivalence

$$
\alpha=e_{0}+e_{1} x+\cdots+e_{k-1} x^{k-1}+\sum_{j=k}^{n} r_{j} x^{j},
$$

where $e_{i} \in\left(1-e_{i-1}\right) \ldots\left(1-e_{0}\right) I\left(1-e_{0}\right) \ldots\left(1-e_{i-1}\right)$ for $i=1, \ldots, k-1$ and $r_{j} \in\left(1-e_{k-1}\right) \ldots\left(1-e_{0}\right) I(1-$ $\left.e_{0}\right) \ldots\left(1-e_{k-1}\right)$ for $j=k, \ldots, n$. When we take $k=n$, theorem will be proved. In this case we have that

$$
\alpha=e_{0}+e_{1} x+\cdots+e_{k-1} x^{k-1}+r_{n} x^{n},
$$

where $e_{i} \in\left(1-e_{i-1}\right) \ldots\left(1-e_{0}\right) I\left(1-e_{0}\right) \ldots\left(1-e_{i-1}\right)$ for $i=1, \ldots, n-1$ and $r_{n} \in h I h$ with $h:=\left(1-e_{0}\right) \ldots\left(1-e_{n-1}\right)$ . It is known $h R h$ unit-regular by [4, Corollary 4.7], so there exists a unit $u \in h R h$ with inverse $v$ and an idempotent $e_{n} \in h R h$ such that $r_{n}=u e_{n}$. We have $e_{n}=v r_{n} \in h I h$, because $r_{n} \in h I h$. Clearly, $\left(e_{0}+\cdots+e_{n-1}\right)+v$ is a unit in $R$ and

$$
\left(e_{0}+\cdots+e_{n-1}+v\right) \alpha=e_{0}+e_{1} x+\cdots+e_{n-1} x^{n-1}+e_{n} x^{n} .
$$

Proof of Claim : We prove it by induction on $k$.

Let $k=1$ and $\alpha=r_{0}+r_{1} x+\cdots+r_{n} x^{n} \in T$. Every $r_{0}$ can be written as a product of unit and an idempotent because $R$ is unit regular. Hence, up to equivalence, left multiplying $\alpha$ by a suitable unit of $R$, we can assume that $r_{0}=e_{0}$ is an idempotent. Because

$$
\left(1-\left(1-e_{0}\right) r_{1} x\right) \alpha\left(1-r_{1} x\right)=e_{0}+\left(1-e_{0}\right) r_{1}\left(1-e_{0}\right) x+\ldots
$$


where both $1-\left(1-e_{0}\right) r_{1} x$ and $1-r_{1} x$ are units of $T$, we can further assume that $r_{1} \in\left(1-e_{0}\right) I\left(1-e_{0}\right)$. Now

$$
\left(1-\left(1-e_{0}\right) r_{2} x^{2}\right) \alpha\left(1-r_{2} x^{2}\right)=e_{0}+r_{1} x+\left(1-e_{0}\right) r_{2}\left(1-e_{0}\right) x^{2}+\ldots
$$

where both $1-\left(1-e_{0}\right) r_{2} x^{2}$ and $1-r_{2} x^{2}$ are units of $T$, so we can assume that $r_{2} \in\left(1-e_{0}\right) I\left(1-e_{0}\right)$. A simple induction shows that

$$
\alpha=e_{0}+r_{1} x+r_{2} x^{2}+\cdots+r_{n} x^{n} r_{i} \in\left(1-e_{0}\right) I\left(1-e_{0}\right) \text { for } i=1, \ldots, n .
$$

So the case $k=1$ is proved. Set

Assume that $(*)$ holds for a fixed integer $k$ with $1<k<n$. It is clear that $e_{0}, \ldots, e_{k-1}$ are orthogonal idempotents.

$f_{k-1}:=\left(1-e_{0}\right) \ldots\left(1-e_{k-1}\right) \in I$ and $g_{k-1}:=e_{0}+\cdots+e_{k-1} \in R$. Then $f_{k-1}$ and $g_{k-1}$ are orthogonal idempotents and $f_{k-1}+g_{k-1}=1$. Because $f_{k-1} R f_{k-1}$ is unit regular by [4, Corollary 4.7], write $r_{k}=u e_{k}$ where $e_{k}$ is an idempotent element in $f_{k-1} R f_{k-1}$ and $u$ is an unit element of $f_{k-1} R f_{k-1}$ with inverse $v$. Then $e_{k}=v r_{k} \in f_{k-1} I f_{k-1}$, since $r_{k} \in f_{k-1} I f_{k-1}$. Then $g_{k-1}+v$ is a unit of $R$ with inverse $g_{k-1}+u$. Since

$$
\left(g_{k-1}+v\right) \alpha=e_{0}+e_{1} x+\cdots+e_{k} x^{k}+\sum_{j=k+1}^{n} v r_{j} x^{j},
$$

up to equivalence, we can assume that

$$
\alpha=e_{0}+e_{1} x+\cdots+e_{k} x^{k}+\sum_{j=k+1}^{n} r_{j} x^{j}
$$

where $e_{k}^{2}=e_{k} \in f_{k-1} I f_{k-1}$ and $r_{j} \in f_{k-1} I f_{k-1}$ for $j=k+1, \ldots, n$. Now

$$
\alpha^{\prime}:=\left(1-r_{k+1} x\right) \alpha=e_{0}+e_{1} x+\cdots+e_{k} x^{k}+r_{k+1}\left(1-e_{k}\right) x^{k+1}+\sum_{j=k+2}^{n} r_{j}^{\prime} x^{j},
$$

where $r_{k+1}, r_{k+2}^{\prime}, \ldots, r_{n}^{\prime} \in f_{k-1} I f_{k-1}$. Set $r_{k+1}^{\prime}:=r_{k+1}\left(1-e_{k}\right)$. Then compute,

$$
\left(1-\left(1-e_{k}\right) r_{k+1}^{\prime} x\right) \alpha^{\prime}\left(1-r_{k+1}^{\prime} x\right)=\sum_{i=0}^{k} e_{i} x^{i}+\sum_{j=k+1}^{n} r_{j}^{\prime \prime} x^{j},
$$

where

$$
\begin{aligned}
r_{k+1}^{\prime \prime} & =r_{k+1}^{\prime}-e_{k} \sigma^{k}\left(r_{k+1}^{\prime}\right)-\left(1-e_{k}\right) r_{k+1}^{\prime} e_{k} \\
& =e_{k}\left(r_{k+1}^{\prime}-\sigma^{k}\left(r_{k+1}^{\prime}\right)\right)+\left(1-e_{k}\right) r_{k+1}^{\prime}\left(1-e_{k}\right) \\
& =e_{k}\left(r_{k+1}-\sigma^{k}\left(r_{k+1}\right)\right)\left(1-e_{k}\right)+\left(1-e_{k}\right) r_{k+1}^{\prime}\left(1-e_{k}\right) \\
& =\left(1-e_{k}\right) r_{k+1}^{\prime}\left(1-e_{k}\right) \in\left(1-e_{k}\right) f_{k-1} I f_{k-1}\left(1-e_{k}\right)
\end{aligned}
$$

since $e_{k}\left(r_{k+1}-\sigma^{k}\left(r_{k+1}\right)\right)\left(1-e_{k}\right)$ by Proposition 1, and where all $r_{i}^{\prime \prime} \in f_{k-1} I f_{k-1}$ for $i \geq k+2$.

We set $f_{i}:=\left(1-e_{0}\right) \ldots\left(1-e_{i}\right)$ for $i=0,1, \ldots, k$. Up to equivalence we may assume that

$$
\alpha=\sum_{i=0}^{k} e_{i} x^{i}+r_{k+1} x^{k+1}+\sum_{j=k+2}^{n} r_{j} x^{j},
$$

where $e_{i}=e_{i}^{2} \in f_{i-1} I f_{i-1}$ for $i=1, \ldots, k$ and where $r_{k+1} \in f_{k} I f_{k}, r_{j} \in f_{k-1} I f_{k-1} j=k+2, \ldots, n$. Then compute

$$
\begin{aligned}
\alpha^{\prime} & =\left(1-r_{k+2} x^{2}\right) \alpha \\
& =\sum_{i=0}^{k} e_{i} x^{i}+r_{k+1} x^{k+1}+\sum_{j=k+2}^{n} r_{j}^{\prime} x^{j},
\end{aligned}
$$

where $r_{j}^{\prime} \in f_{i-1} I f_{i-1}$ for $j>k+2$ and where $r_{k+2}^{\prime}=r_{k+2}\left(1-e_{k}\right)$. Then compute

$$
\left(1-\left(1-e_{k}\right) r_{k+2}^{\prime} x^{2}\right) \alpha^{\prime}\left(1-r_{k+2}^{\prime} x^{2}\right)=\sum_{i=0}^{k} e_{i} x^{i}+r_{k+1} x^{k+1}+\sum_{j=k+2}^{n} r_{j}^{\prime \prime} x^{j},
$$


where

$$
\begin{aligned}
r_{k+2}^{\prime \prime} & =r_{k+2}^{\prime}-e_{k} \sigma^{k}\left(r_{k+2}^{\prime}\right)-\left(1-e_{k}\right) r_{k+2}^{\prime} e_{k} \\
& =e_{k}\left(r_{k+2}^{\prime}-\sigma^{k}\left(r_{k+2}^{\prime}\right)\right)+\left(1-e_{k}\right) r_{k+2}^{\prime}\left(1-e_{k}\right) \\
& =e_{k}\left(r_{k+2}-\sigma^{k}\left(r_{k+2}\right)\right)\left(1-e_{k}\right)+\left(1-e_{k}\right) r_{k+2}^{\prime}\left(1-e_{k}\right) \\
& =\left(1-e_{k}\right) r_{k+2}^{\prime}\left(1-e_{k}\right) \in\left(1-e_{k}\right) f_{k-1} I f_{k-1}\left(1-e_{k}\right)=f_{k} I f_{k}
\end{aligned}
$$

since $e_{k}\left(r_{k+2}-\sigma^{k}\left(r_{k+2}\right)\right)\left(1-e_{k}\right)$ by Proposition 1 , and where all $r_{i}^{\prime \prime} \in f_{k-1} I f_{k-1}$ for $i \geq k+3$. Repeating analogous arguments, up to equivalence we may assume that

$$
\alpha=e_{0}+e_{1} x+\cdots+e_{k} x^{k}+\sum_{j=k+1}^{n} r_{j} x^{j},
$$

where $r_{j} \in f_{k} I f_{k}$ for $j=k+1, \ldots, n$. So we complete the inductive step and we are done.

Corollary 3. Let $I$ be an ideal of a unit regular ring $R$ and let $\sigma: R \rightarrow R$ is a ring endomorphism such that $\sigma(e)=e$ for all $e^{2}=e \in R$. Then $T$ is a left morphic ring for each $n \geq 0$.

Proof. We will show arbitrary $\alpha \in T$ is left morphic in $T=[R ; I, \sigma, n]$. Let $\beta=\sum_{i=0}^{n} b_{i} x^{i} \in T$, where $b_{0}=$ $\left(1-e_{0}\right)\left(1-e_{1}\right) \ldots\left(1-e_{n}\right)=1-e_{0}-e_{1}-\cdots-e_{n}$ and $b_{i}=e_{n-i}$ for $i=1, \ldots, n$.

Claim : $T \alpha=\mathbf{l}_{T} \beta$ and $T \beta=\mathbf{l}_{T} \alpha$. By Theorem 2, $\alpha$ is equivalent to $\gamma:=e_{0}+e_{1} x+\cdots+e_{n} x^{n}$, where $e_{0}^{2}=e_{0} \in R$ and $e_{i}^{2}=e_{i} \in\left(1-e_{i-1}\right) \ldots\left(1-e_{0}\right) I\left(1-e_{0}\right) \ldots\left(1-e_{i-1}\right)$ for $i=1, \ldots, n$.

Given $\lambda=\sum_{i=0}^{n} r_{i} x^{i} \in T$ with $r_{0} \in R e_{0}$ and $r_{i} \in I \sum_{j=0}^{i} e_{j}$, let $\gamma=\sum_{i=0}^{n} a_{i} x^{i} \in T$, where

$$
\begin{aligned}
a_{0} & =r_{0} e_{0}+r_{1} e_{1}+\cdots+r_{n} e_{n} \\
a_{1} & =r_{1} e_{0}+r_{2} e_{1}+\cdots+r_{n} e_{n-1} \\
& \vdots \\
a_{i} & =r_{i} e_{0}+r_{i+1} e_{1}+\cdots+r_{n} e_{n-i} \\
& \vdots \\
a_{n} & =r_{n} e_{0}
\end{aligned} .
$$

Then $\lambda=\gamma \alpha \in T \alpha$.

For any $\omega=\sum_{i=0}^{n} a_{i} x^{i} \sum_{j=0}^{n} e_{j} x^{j} \in T \alpha$, the coefficient of $x^{k}$, is

$$
\begin{aligned}
& \sum_{i=0}^{k} a_{i} \sigma^{i}\left(e_{k-i}\right)=\sum_{i=0}^{n} a_{i}\left(e_{k-i}\right)=a_{0} e_{k}+a_{1} e_{k-1}+\cdots+a_{k} e_{0} \\
& =\left(a_{0} e_{k}+a_{1} e_{k-1}+\cdots+a_{k} e_{0}\right)\left(e_{0}+e_{1}+\cdots+e_{k}\right) \in I\left(e_{0}+e_{1}+\cdots+e_{k}\right) .
\end{aligned}
$$

Hence,

Similarly,

$$
\begin{aligned}
T \alpha= & \left\{r_{0}+r_{1} x+\cdots+r_{n-1} x^{n}: r_{0} \in R e_{0}, r_{1} \in I\left(e_{0}+e_{1}\right), \ldots,\right. \\
& \left.r_{n} \in I\left(e_{0}+e_{1}+\cdots+e_{n}\right)\right\}
\end{aligned}
$$

$$
\begin{aligned}
T \beta= & \left\{r_{0}+r_{1} x+\cdots+r_{n-1} x^{n}: r_{0} \in R\left(1-e_{0}-e_{1}-\cdots-e_{n}\right),\right. \\
& \left.r_{1} \in I\left(1-e_{0}-e_{1}-\cdots-e_{n-1}\right), \ldots, r_{n} \in I\left(1-e_{0}\right)\right\} .
\end{aligned}
$$

For any $\gamma=\sum_{i=0}^{n} a_{i} x^{i} \in T$, we have $\gamma \in \mathbf{l}_{T}(\alpha)$ if and only if

$$
\begin{aligned}
& a_{0} e_{0}=0, \\
& a_{0} e_{1}+a_{1} \sigma\left(e_{0}\right)=0, \\
& a_{0} e_{2}+a_{1} \sigma\left(e_{1}\right)+a_{2} \sigma^{2}\left(e_{0}\right)=0, \\
& \vdots \\
& a_{0} e_{n}+a_{1} \sigma\left(e_{n-1}\right)+\cdots+a_{n} \sigma^{n}\left(e_{0}\right)=0,
\end{aligned}
$$

but since $\sigma(e)=0$ for all idempotens, we have,

$$
\begin{aligned}
& a_{0} e_{0}=0, \\
& a_{0} e_{1}+a_{1} e_{0}=0, \\
& a_{0} e_{2}+a_{1} e_{1}+a_{2} e_{0}=0, \\
& \vdots \\
& a_{0} e_{n}+a_{1} e_{n-1}+\cdots+a_{n} e_{0}=0,
\end{aligned}
$$


if and only if

$$
\begin{aligned}
a_{0} e_{i} & =0 \quad(0 \leq i \leq n) \\
a_{1} e_{i} & =0 \quad(0 \leq i \leq n-1) \\
& \vdots \\
a_{j} e_{i} & =0 \quad(0 \leq i \leq n-j) \\
& \vdots \\
a_{n} e_{0} & =0
\end{aligned}
$$

if and only if $a_{0} \in R\left(1-\sum_{i=0}^{n} e_{i}\right)$ and $a_{j} \in I\left(1-\sum_{i=0}^{n-j} e_{i}\right)$ for any $j$ with $1 \leq j \leq n$. Hence $\mathbf{l}_{T}(\alpha)=T \beta$. Using similar argument one can have $\mathbf{l}_{T}(\beta)=T \alpha$. So $T$ is left morphic.

Corollary 4. [8, Corollary 3] If $R$ is a unit regular ring and $\sigma: R \rightarrow R$ is a ring endomorphism such that $\sigma(e)=e$ for all $e^{2}=e \in R$. Then $R[x ; \sigma] /\left(x^{n+1}\right)$ is a left morphic ring for all $n \geq 0$.

Proof. Let take $I=R$ in the previous corollary then the result follows.

\section{The ring $(R, I)(x) /\left(x^{n+1}\right)$ is centrally morphic}

Remark 5. If $R$ is a unit-regular ring and if $\alpha \in S=(R, I)(x) /\left(x^{n+1}\right)$ where $n \geq 0$, then by Theorem 2 there exist a sequence of orthogonal idempotents $e_{0}, e_{1}, \ldots, e_{n}$, where $e_{0} \in R, e_{1}, \ldots, e_{n} \in I$, and units $u, v \in U(S)$ such that $\alpha=$ $v\left(e_{0}+e_{1} x+\ldots+e_{n} x^{n}\right) u$. Thus, $\alpha S=v\left(e_{0}+e_{1} x+\ldots+e_{n} x^{n}\right) S$ and $S \alpha=S\left(e_{0}+e_{1} x+\ldots+e_{n} x^{n}\right) u$.

For $\alpha=\sum_{i=0}^{n} a_{i} x^{i} \in S$, let

$$
\alpha^{\circ}=\left(1-a_{0}-\ldots-a_{n}\right)+a_{n} x+\ldots+a_{1} x^{n} .
$$

Note that $\left(\alpha^{\circ}\right)^{\circ}=\alpha$ for all $\alpha \in S$

Lemma 6. Let $R$ be a ring and let $\alpha=\sum_{i=0}^{n} e_{i} x^{i} \in S=(R, I)(x) /\left(x^{n+1}\right)$ where $e_{0}, e_{1}, \ldots, e_{n}$ is a sequence of orthogonal idempotents such that $e_{0} \in R$ and $e_{1}, \ldots, e_{n} \in I$. Then

$$
S(\alpha)=\mathbf{l}\left(\alpha^{\circ}\right) \text { and } S\left(\alpha^{\circ}\right)=\mathbf{l}(\alpha) .
$$

Proof. An easy calculation shows that

$$
S \alpha=R e_{0}+I\left(e_{0}+e_{1}\right) x+\cdots+I\left(e_{0}+\cdots+e_{n}\right) x^{n}=\mathbf{l}\left(\alpha^{\circ}\right) .
$$

Since $\left(1-e_{0}-\cdots-e_{n}\right), e_{1}, \ldots, e_{n}$ is a sequence of orthogonal idempotents such that $e_{1}, \ldots, e_{n} \in I$ and $\left(1-e_{0}-\right.$ $\left.\cdots-e_{n}\right) \in R$, the second equality follows.

For the next theorem, its proof is a modification of [9, Theorem 20].

Theorem 7. Let $I$ be an ideal of a ring $R$ and let $\sigma: R \rightarrow R$ is a ring endomorphism such that $\sigma(e)=e$ for all $e^{2}=e \in R$. $R$ is strongly regular if and only if $S$ is left centrally morphic for every $n \geq 0$.

Proof. Assume that $R$ is a unit strongly regular ring and let $\alpha \in S=(R, I)(x) /\left(x^{n+1}\right)$. By Theorem 2, there exists orthogonal idempotents $e_{0}, e_{1}, \ldots, e_{n}$, where $e_{1}, \ldots, e_{n} \in I$ and $e_{0} \in R$ such that $\alpha$ is equivalent to $\beta:=$ $e_{0}+e_{1} x+\ldots+e_{n} x^{n} \in S$. By Lemma $6, S \beta=l\left(\beta^{0}\right)$ and $S \beta^{0}=l(\beta)$. Since $R$ is strongly regular all idempotents of $R$ are central. So $\beta$ and $\beta^{0}$ are central in $S$. Then there exists $u, v \in U(S)$ such that $\alpha=u \beta v=(u v) \beta$. It follows that $S \alpha=S \beta=l\left(\beta^{0}\right)$ and $l(\alpha)=l(\beta)=S \beta^{0}$. So $S$ is left centrally morphic.

Let $a \in R$. Since $a$ is left morphic in $R$ by Lemma 15, we have $R a=l(b)$ for some $b \in R$. Let $\alpha=b x^{n} \in S$. Then there exists $\beta=\sum_{i=0}^{n} b_{i} x^{i} \in C(S)$ such that $l(\alpha)=S \beta$. We have $b_{i} \in C(I)$ for $i=1, \ldots, n$ and $b_{0} \in C(R)$ because $\beta \in C(S)$. By computation, one can obtain

$$
l(\alpha)=l(b)+I x+\ldots+I x^{n}
$$


and $S \beta=\left\{r_{0} b_{0}+\left(r_{0} b_{1}+r_{1} b_{0}\right) x+\ldots+\left(r_{0} b_{n}+\ldots+r_{n} b_{0}\right) x^{n}: r_{i} \in R\right.$ for $1 \leq i \leq n$ and $\left.r_{0} \in R\right\}$. Hence there exists $r_{0} \in R, r_{1} \in I$ such that $0=r_{0} b_{0}$. Also $S \beta=l(\alpha) \subseteq l(b)+R x+\ldots+R x^{n}$ we have $1=r_{0} b_{1}+r_{1} b_{0}$. So $b_{0}=b_{0}\left(r_{0} b_{1}+r_{1} b_{0}\right)=b_{0} r_{0} b_{1}+b_{0} r_{1} b_{0}=r_{0} b_{0} b_{1}+b_{0} r_{1} b_{0}=b_{0} r_{1} b_{0}$. Therefore $b_{0}$ is regular in $R$. But from $l(\alpha)=S \beta$ it follows that $R b_{0}=l(b)$. Since $l(b)=R a$ we have $R a=R b_{0}$ is an ideal of $R$. Thus we have proved that $R$ is regular and every principal left ideal of $R$ is an ideal. Hence $R$ is strongly regular by [4, Theorem 3.2].

Corollary 8. [9, Theorem 20] Let $n \geq 1$ be an integer. Then $R$ is strongly regular if and only if $R[x] /\left(x^{n+1}\right)$ is a left centrally morphic ring.

Proof. Let $I=R$, then proof is by previous theorem.

\section{The ring $(R, I)(x) /\left(x^{n+1}\right)$ is left quasi-morphic}

First we fix some notation. Following Herbera [5], we define set $E$ as following:

$$
E=\left\{e(x) \in(R, I)[[x]]: e(x)=e+\sum_{i=1}^{\infty}(1-e) a_{i} e x^{i} \text { where } e^{2}=e \in R \text { and } a_{i} \in I \text { for } i=1, \ldots\right\}
$$

Fix an integer $n$ and $(R, I)[[x]] /\left(x^{n+1}\right) \cong(R, I)(x) /\left(x^{n+1}\right)$. For any $\alpha=\sum_{i=0}^{\infty} a_{i} x^{i} \in(R, I)[[x]]$, let $\bar{\alpha}=$ $\sum_{i=0}^{n} a_{i} x^{i}$ be the image of $\alpha$. We let

$$
\bar{E}=\{\overline{e(x)}: e(x) \in E\} .
$$

The following two lemmas are a generalization of [5, Lemma 1.3 and Lemma 1.4]. The proofs are similar to [5] but for the sake of completeness, we write them again.

Remark 9. (1) The elements of $E$ are idempotents of $(R, I)[[x]]$.

(2) Let $e(x)=e+\sum_{i=1}^{\infty}(1-e) a_{i} e x^{i} \in E$, then $\mathbf{r}_{(R, I)[[x]]}(e(x))=\mathbf{r}_{R}(e)(R, I)[[x]]$

Lemma 10. Let $R$ be a regular ring, $I$ be an ideal of $R$ and $a(x) \in(R, I)[[x]]$. Then there exists power series $e(x) \in E$ and $a^{\prime}(x)$ such that

$$
a(x)(R, I)[[x]]=e(x)(R, I)[[x]]+x a^{\prime}(x)(R, I)[[x]] \text { and } e(x) a^{\prime}(x)=0 .
$$

Moreover,

$$
1_{R}(a(x)) \subseteq 1_{R}(e(x)) \cap 1_{R}\left(a^{\prime}(x)\right) .
$$

Proof. If the zero degree term of $a(x)$ is zero then the proof is clear. Assume that

$$
a(x)=a_{0}+x \widetilde{a}(x)
$$

with $0 \neq a_{0} \in R$ and $\widetilde{a}(x) \in I(x) /\left(x^{n+1}\right)$. Since $R$ is regular there exists an element $t \in R$ such that $a_{0} t a_{0}=a_{0}$. So $a_{0} t=e$ and $t a_{0}=f$ are idempotent elements of $R$. Then

$$
\begin{aligned}
a(x)(R, I)[[x]] & =a(x) f(R, I)[[x]]+a(x)(1-f)(R, I)[[x]] \\
& =a(x) f(R, I)[[x]]+x \widetilde{a}(x)(1-f)(R, I)[[x]] .
\end{aligned}
$$

Moreover,

$$
\begin{aligned}
a(x) f(R, I)[[x]] & =(e a(x) f+(1-e) \widetilde{a}(x) f x)(R, I)[[x]] \\
& =(e a(x) f+(1-e) \widetilde{a}(x) f x) t e(R, I)[[x]] .
\end{aligned}
$$

$e a(x) f t e+1-e$ is a unit element of $(R, I)[[x]]$ because $e a_{0} f t e=e$. Let $u(x)$ be inverse of $e a(x) f t e+1-e$. Also note that $u(x)=e u(x) e+1-e$. Thus $e a(x) f t e=(e u(x) e)^{-1}$ is a unit of $e(R, I)[[x]] e$. So we have

$$
a(x) f(R, I)[[x]]=e(x)(R, I)[[x]],
$$

where

$$
e(x)=(e a(x) f+(1-e) a(x) f) t e(e u(x) e+(1-e))=e+\sum_{i=1}^{\infty}(1-e) b_{n} e x^{n},
$$


for suitable $b_{n} \in I$. By definition of the set $E, e(x)$ is an element of the set $E$. Hence,

$$
\begin{aligned}
a(x)(R, I)[[x]] & =e(x)(R, I)[[x]]+x \widetilde{a}(x)(1-f)(R, I)[[x]] \\
& =e(x)(R, I)[[x]]+x(1-e(x)) \widetilde{a}(x)(1-f)(R, I)[[x]] .
\end{aligned}
$$

If we choose $a^{\prime}(x):=(1-e(x)) \widetilde{a}(x)(1-f)(R, I)[[x]]$, then we are done. For the moreover part, it suffices to show that $l_{R}(a(x)) \subseteq l_{R}(e(x))$. If $r \in l_{R}(a(x))$ then $r e=0$, and so $r(1-e)=r$. But $e(x)=(e a(x) f+(1-e) a(x) f) t e(e u(x) e+$ $(1-e))$, so we have $r e(x)=0$.

Lemma 11. Let $R$ be a regular ring, $I$ be an ideal of $R$ and $a(x) \in(R, I)[[x]]$. Then there exits sequence of idempotents $e_{i}(x) \in E$ such that, for any $n \geq 0$, there exits $a_{n}^{\prime}(x) \in(R, I)[[x]]$ which satisfies

$$
a(x)(R, I)[[x]]=\left(\sum_{i=0}^{n} e_{i}(x) x^{i}\right)(R, I)[[x]]+a_{n}^{\prime}(x) x^{n+1}(R, I)[[x]] .
$$

Moreover,

(i) $e_{i}(x) e_{j}(x)=0$ for any $j>i \geq 0$, and

(ii) for every $0 \leq i \leq n, e_{i}(x) a_{n}^{\prime}(x)=0$.

Proof. We will proof it by induction on $n$. For the case $n=0$, there is no need to prove because of Lemma 10 . Assume $n \geq 1$ and statement is true for $n-1$. Then

$$
a(x)(R, I)[[x]]=\left(\sum_{i=0}^{n-1} e_{i}(x) x^{i}\right)(R, I)[[x]]+a_{n-1}^{\prime}(x) x^{n}(R, I)[[x]],
$$

and this decomposition satisfies $(i)$ and $(i i)$. By applying Lemma 10 to $a_{n-1}^{\prime}(x)$ we have the equality:

$$
a_{n-1}^{\prime}(x) x^{n}(R, I)[[x]]=\left(e_{n}(x) x^{n}\right)(R, I)[[x]]+a_{n}^{\prime}(x) x^{n+1}(R, I)[[x]],
$$

with $e_{n}(x) \in E, e_{n}(x) a_{n}^{\prime}(x)=0$ and $l_{R}\left(a_{n-1}^{\prime}(x)\right) \subseteq l_{R}\left(e_{n}(x)\right) \cap l_{R}\left(a_{n}^{\prime}(x)\right)$. Since $e_{i}(x) a_{n-1}^{\prime}(x)=0$, for $0 \leq i \leq n-1$, by Remark 9, this happens iff $e_{i} a_{n-1}^{\prime}(x)=0$, where $e_{i}=e_{i}^{2} \in R$ is the term of zero degree of $e_{i}(x)$. Thus $e_{i}(x) e_{n}(x)=0$ and $e_{i}(x) a_{n}^{\prime}(x)=0$, for $0 \leq i \leq n-1$.

Proposition 12. Let $R$ be a regular ring and let $S=(R, I)(x) /\left(x^{n+1}\right)$ where $n \geq 0$. For $\alpha \in S$ the followings are true:

(i) There exits a sequence of orthogonal idempotents $e_{0}, e_{1}, \ldots, e_{n} \in R$ and $u \in U(S)$ such that $S \alpha=S\left(e_{0}+e_{1} x+\cdots+\right.$ $\left.e_{n} x^{n}\right) u$.

(ii) There exits a sequence of orthogonal idempotents $f_{0}, f_{1}, \ldots, f_{n} \in R$ and $v \in U(S)$ such that $\alpha S=v\left(f_{0}+f_{1} x+\cdots+\right.$ $\left.f_{n} x^{n}\right) S$.

Proof. By symmetry it is enough to prove one of the statement. We will prove the statement $(i i)$.

In order to apply Lemma 11, we will think $S$ as $(R, I)[[x]] /\left(x^{n+1}\right)$. Modulo the ideal $\left(x^{n+1}\right)$, the equality in Lemma 11 becomes

$$
\alpha S=\left(\sum_{k=0}^{n} \overline{e_{k}(x)} x^{k}\right) S
$$

where $\overline{e_{k}(x)} \in \bar{E}$ and $\overline{e_{i}(x)} \cdot \overline{e_{j}(x)}=0$ whenever $j>i \geq 0$. We will proceed just as in [9, Proposition 1]. For each $k$ with $0 \leq k \leq n$, let $\overline{e_{k}(x)}=e_{k}+\sum_{i=1}^{n}\left(1-e_{k}\right) a_{i}^{(k)} e_{k} x^{i}$. It follows that $e_{i} e_{j}=0$ whenever $j>i \geq 0$. Now we will use the same technique in [5, Corollary 1.7]. Let $\overline{e_{k}(x)}=e_{k}+\sum_{i=1}^{n}\left(1-e_{k}\right) a_{i}^{(k)} e_{k} x^{i}$, for each $k$ with $0 \leq k \leq n$. Then we have $e_{i} e_{j}=0$, whenever $j>i \geq 0$. Hence $\sum_{k=0}^{n} e_{k} R=\bigoplus_{k=0}^{n} e_{k} R$. By hypothesis we can write $\bigoplus_{k=0}^{n} e_{k} R=e R$ where $e$ is an idempotent element of $R$. Define $h_{i}: \bigoplus_{k=0}^{n} e_{k} R \rightarrow R$ by $h_{i}\left(\sum_{k=0}^{n} e_{k} r_{k}\right)=\sum_{k=0}^{n}\left(1-e_{k}\right) a_{i}^{(k)} e_{k} r_{k}$ which is a left multiplication by $b_{i}=h_{i}(e)$, for each $i$ with $1 \leq i \leq n$. So $b_{i} e_{k}=h_{i}\left(e_{k}\right)=\left(1-e_{k}\right) a_{i}^{(k)} e_{k}$. Define $v$ as following:

$$
v:=1+\sum_{i=1}^{n} b_{i} x^{i}
$$

Then $v \in U(S)$ and $v\left(\sum_{k=0}^{n} e_{k} x^{k}\right)=\sum_{k=0}^{n} \overline{e_{k}(x)} x^{k}$. Sequence of orthogonal idempotents $\left\{f_{k}\right\}_{k=0}^{n}$ can be constructed such as :

$$
f_{0}=e_{0} \text { and } f_{k}=e_{k}\left(1-f_{0}-\cdots-f_{k-1}\right) \text { for } k=1, \ldots, n
$$


By [5, Remark 1.6], the $R$-module epimorphism $g: \bigoplus_{k=0}^{n} R e_{k} \rightarrow \bigoplus_{k=0}^{n} R f_{k}$ which is given by $g\left(\sum_{k=0}^{n} r_{k} e_{k}\right)=$ $\sum_{k=0}^{n} r_{k} f_{k}$ is an isomorphism. Write $\bigoplus_{k=0}^{n} R e_{k}=R a$ and $\bigoplus_{k=0}^{n} R f_{k}=R b$, where $a$ and $b$ are idempotents of $R$. Say $c=g(a)$ and $d=g^{-1}(b)$. Then $g$ and $g^{-1}$ are the right multiplication by $c$, and $d$, respectively. Hence

$$
\sum_{k=0}^{n} e_{k} x^{k}=\sum_{k=0}^{n} g^{-1}\left(f_{k}\right) x^{k}=\sum_{k=0}^{n} f_{k} d x^{k}=\left(\sum_{k=0}^{n} f_{k} x^{k}\right) d
$$

and

$$
\sum_{k=0}^{n} f_{k} x^{k}=\sum_{k=0}^{n} g\left(e_{k}\right) x^{k}=\sum_{k=0}^{n} e_{k} c x^{k}=\left(\sum_{k=0}^{n} e_{k} x^{k}\right) c .
$$

So $\left(\sum_{k=0}^{n} e_{k} x^{k}\right) S=\left(\sum_{k=0}^{n} f_{k} x^{k}\right) S$. Hence $\alpha S=v\left(\sum_{k=0}^{n} e_{k} x^{k}\right) S=v\left(\sum_{k=0}^{n} f_{k} x^{k}\right) S$.

Theorem 13. Let $R$ be a regular ring and let $n \geq 0$. Then $S=(R, I)(x) /\left(x^{n+1}\right)$ is a quasi-morphic ring.

Proof. By symmetry, we only show that $S$ is a left quasi morphic ring. Let $\alpha \in S$. By Proposition 12,

$$
S \alpha=S\left(e_{0}+e_{1} x+\ldots+e_{n} x^{n}\right) u
$$

and

$$
\alpha S=v\left(f_{0}+f_{1}+\ldots+f_{n} x^{n}\right) S,
$$

where $u, v$ are unit elements of $S$ and $\left\{e_{i}\right\}_{i=0}^{n},\left\{f_{i}\right\}_{i=0}^{n}$ are sequences of orthogonal idempotents of $R$. Let $\beta=$ $\sum_{i=0}^{n} e_{i} x^{i}$ and $\gamma=\sum_{i=0}^{n} f_{i} x^{i}$. Then, by [9, Lemma 3],

$$
\begin{gathered}
S \alpha=(S \beta) u=l\left(\beta^{0}\right) u=l\left(u^{-1} \beta^{0}\right), \\
l(\alpha)=l(v \gamma)=l(\gamma) v^{-1}=\left(S \gamma^{0}\right) v^{-1}=S\left(\gamma^{0} v^{-1}\right) .
\end{gathered}
$$

So $\alpha$ is a left quasi-morphic in $S$.

Corollary 14. If $R$ is regular and $n \geq 0$, then the matrix rings over $(R, I)(x) /\left(x^{n+1}\right)$ are all quasi-morphic.

Proof. If $R$ is regular then $M_{k}(R)$ is regular for each $k \geq 1$. So $M_{k}\left((R, I)(x) /\left(x^{n+1}\right)\right) \cong M_{k}(R, I)(x) /\left(x^{n+1}\right)$ is quasi-morphic by Theorem 13.

The following theorem generalizes [9, Lemma 10].

Lemma 15. Let $n \geq 0$ be an integer. If $S=(R, I)(x) /\left(x^{n+1}\right)$ is left quasi-morphic (resp., left morphic), then so is $R$.

Proof. Let $a \in R$ and let $\alpha=a \in S$. Since $\alpha$ is left quasi-morphic in $S, S \alpha=\mathbf{l}(\beta)$ and $\mathbf{l}(\alpha)=S \gamma$, where $\beta=\sum_{i=0}^{n} b_{i} x^{i}$ and $\gamma=\sum_{i=0}^{n} c_{i} x^{i}$ inS. But

$$
\begin{gathered}
\mathbf{l}(\alpha)=\mathbf{l}(a)+\mathbf{l}(a) x+\cdots+\mathbf{l}(a) x^{n} \text { and } \\
S \gamma=\left\{r_{0} c_{0}+\left(r_{0} c_{1}+r_{1} c_{0}\right) x+\cdots+\left(r_{0} c_{n}+r_{1} c_{n-1}+\cdots+r_{n} c_{0}\right) x^{n}: r_{0} \in R r_{1}, \ldots, r_{n} \in I\right\} .
\end{gathered}
$$

So it follows from $\mathrm{l}(\alpha)=S \gamma$ that $\mathbf{l}(a)=R c_{0}$. On the other hand, $\alpha \beta=0$ clearly implies that $R a \subseteq \mathbf{l} b_{0}$. Moreover,

$$
\left(\mathbf{l}\left(b_{0}\right) \cap \cdots \cap \mathbf{l}\left(b_{n}\right)\right)+\left(\mathbf{l}\left(b_{0}\right) \cap \cdots \cap \mathbf{l}\left(b_{n-1}\right)\right) x+\cdots+\mathbf{l}\left(b_{0}\right) x^{n}
$$

$\mathbf{l}(\beta)=S \alpha=R a+\operatorname{Iax}+\cdots+\operatorname{Iax}^{n}$.

So $\mathbf{l}\left(b_{0}\right) \subseteq R a$. Hence $R a=\mathbf{l}\left(b_{0}\right)$. So $a$ is left quasi-morphic in $R$. If $\alpha$ is left morphic in $S$, then $\beta$ and $\gamma$ can be chosen to be the same. Thus, $a$ is left morphic in $R$ since $b_{0}=c_{0}$ in this case. 


\section{References}

[1] Camillo V., Nicholson W.K. Quasi-morphic rings, J. Algebra Applications 2007; 6: 789 -799.

[2] Chen J. L. and Zhou Y., Glasg. Math. J. 2005, 47 (1): 139-148.

[3] Ehrlich G., Units and one-sided units in regular rings, Trans. AMS 1976; 216: 81-90.

[4] Goodearl K.R. Von Neumann Regular Rings, Monographs and Studies in Mathematics, Pitman, Boston: Mass.London, 1979.

[5] Herbera D. Bezeout and semiheriditary power series ring, Journal of Algebra 2003; 270: 150-168.

[6] Huang Q., Chen J. Morphic properties of extension rings, Algebra Colloquim 2010; 270:337-344

[7] Lee T.K., Zhou Y. Morphic rings and unit regular rings, J. Pure. Appl. Algebra 2007; 210: 501-510.

[8] Lee T.K., Zhou Y. A theorem on unit regular rings, Canadian Math. Bull 2010; 53: 321-326.

[9] Lee T.K., Zhou Y. Regularity and Morphic property of rings, J. Algebra 2009; 322: 1072-1085.

[10] Nicholson W. K., Sánchez Campós E., Rings with the dual of the isomorphism theorem, J. Algebra 2004; 271: 391-406.

\section{Affiliations}

SERAP ŞAHINKAYA

ADDRESS: Gebze Technical University, Department of Mathematics, Çayirova Campus 41400 Gebze- Kocaeli, Turkey

E-MAIL: ssahinkaya@gtu.edu.tr 